\title{
A New Model of Counterion Condensation in Polyelectrolyte Solutions I. Comparison with Other Condensation Theories and Experimental Data on Counterion Activity Coefficients
}

\author{
Mitsuru SATOH, ${ }^{*}$ Takehito KaWASHIMA, Jiro KomIYAMA, \\ and Toshiro IIJIMA
}

Department of Polymer Science, Tokyo Institute of Technology,

Ookayama, Meguro-ku, Tokyo 152, Japan

(Received April 2, 1987)

\begin{abstract}
A new model of counterion condensation (Intermediate Model, IMM) is proposed as a modification of our corrected previous model (CPM). The theoretical predictions of IMM and CPM for the degree of condensation, $\theta$, and the counterion activity coefficient, $\gamma$, are compared with those of Manning and Iwasa et al. It is found that $\gamma$ 's in the absence of added salts estimated by IMM and CPM for infinitely long polyions are hardly dependent on the polymer concentration, $C_{\mathrm{p}}$, while those for polyions with finite length increase with decreasing $C_{\mathrm{p}}$. It is the latter that is experimentally observed. This fact indicates that polymer models, in which end effects are considered, should be employed to treat dependence of the condensation phenomena on the ionic strength.

KEY WORDS Polyelectrolyte / Counterion Condensation / Activity

Coefficient /
\end{abstract}

Manning's limiting law for counterion condensation in polyelectrolyte solution, which first appeared in $1969,{ }^{1}$ has been applied to many systems including synthetic and biological polymers. ${ }^{2-10}$ In some cases it has shown quantitative agreements with experimental data on the degree of condensation, $\theta$, and the counterion activity coefficient. ${ }^{2-7}$ The limiting law for $\theta$ is expressed as,

$$
\left(1-(v \xi)^{-1}\right) / v, \text { when } \quad v \xi>1
$$

where $\theta$ is the degree of counterion condensation, $v$ is the valence of the counterion, and $\xi$ is the charge density parameter given as $e^{2} / D k T b: e$, the unit charge; $D$, the dielectric constant of the bulk solvent; $k$, the Boltzmann constant; $T$, the Kelvin temperature; $b$, the axial charge spacing on the polyion. Manning has obtained eq 1 also on his two phase model, ${ }^{2}$ which he has further extented to more general systems containing a mixture of dif-

\footnotetext{
* To whom correspondence should be addressed.
}

ferent kind of small ions. ${ }^{11}$ However, if one wants to estimate $\theta$ at a finite ionic strength upon the two phase model, a priori knowledge on the condensed phase volume, $V_{\mathrm{p}}$, is necessary. ${ }^{11}$ In our previous paper, ${ }^{12}$ this difficulty was avoided with a modified model for counterion condensation. It proposed an extention of the two phase model to finite ionic strength (I) and finite polymer length (charge number on a polymer chain, $N$ ). The theoretical predictions on the dependences of $\theta$ and the counterion activity coefficient $\gamma$ on $I$ and $N$ were compared with those of other theories and with experimental data. A major distinction between Manning's two phase model and our previous model lies in the assumptions on the state of the condensed counterions; the former assumes that the condensed ions have translational freedom in the condensed phase, while the latter neglects the mixing entropy term of the condensed counterions, 
thus avoiding introduction of $V_{p}$ term to the free energy calculation. At the same time, however, our model was ambiguous in describing the location of the condensed counterions. Thus the present study reports further extention of our previous model by specifying the binding location to the polyion charge positions. This assumption on the binding location is necessary particularly for more general systems which contain different kind of counterions. For example, extention of the present model to a mixture system of monovalent and divalent counterions is straightforward, ${ }^{13}$ while with Manning's limiting law one needs a rather arbitrary assumption on the volume of the condensed phase. ${ }^{11}$ Besides this modification, we made a correction of our previous treatment on the free energy estimation for polyion-small ion interaction.

The present paper compares the theoretical predictions on $\theta$ and $\gamma$ for the present model, the corrected previous model, and condensation theories of Manning and Iwasa et al. Further, the theoretical $\gamma$ 's are compared with activity coefficients measured for several polyelectrolyte systems (sodium polystyrenesulfonate, PSSNa, sodium poly(L-glutamate), PLGNa, and polyarylamine hydrochloride, PAAHCl) with attention to their dependences on the ionic strength.

\section{MODEL}

We treat a system consisting of polyion, salt composed of common counterion and single valent byion, and solvent (water). We use the following polymer model and assumptions in the free energy calculation.

(1) The polymer chain is represented by a linear array of unit charges with a finite length.

(2) Condensed counterions are bound to $v$ contiguous charged groups on the polyion, reducing each effective charge to $(1-v \theta) e$. Such counterions do not contribute to the mixing entropy of counterions in the bulk phase.

(3) For small ion-polyion interaction, only the long-range electrostatic interaction is taken into account. Effects of the short range interactions are implicitly included in the reduction of the effective polymer charge by the counterion condensation.

(4) Polyion-polyion interactions are neglected.

(5) Effects of changes of solvation (hydration) of small ions and polyions on the condensation are neglected.

(6) The presence of polyion is neglected in the calculation of small ion-small ion electrostatic interactions for added salts.

These assumptions are the same as those of our previous model ${ }^{12}$ except (2) and (6). Polyion chains often assume a coiled conformation, as examples, for systems of high ionic strength or low charge density. However, under the former condition where long range electrostatic interactions are effectively shielded, the models constructed under assumption (1) permits to estimate the remaining short range electrostatic interactions among the real charges. Under the latter condition, the linear charge array model should break down, as it is discussed in the subsequent paper (part II). ${ }^{14}$ Such an estrangement is expedient for discussing the reality of the electrostatic interactions in the relevant systems.

In the previous model, the condensed ions were assumed to stay just around the polyion without specifying the binding position. The present model assumes that upon condensation, non-specific interaction between counterions and polymer charges takes place with the hydration shells intact, and with a uniform lowering of the effective charge by such an interaction. Therefore the present model is different from so-called site-binding model. ${ }^{15,16}$ The latter treats close interaction between a counterion and charged group(s) accompanied by significant dehydration and complete neutralization of the charge like the protonation of weak polyacids. Thus, the 
present model has an intermediate character for the territorial binding and the site binding models.

The last assumption (6) is equivalent to the correction of counterion activity coefficients which has been used in the condensation studies, ${ }^{17,18}$ activity coefficients predicted by condensation theories have been compared with experimental values divided by the activity coefficients in the absence of polyelectrolytes. In the present model, a correction equivalent to this is incorporated in the free energy calculation as shown in the next section. (This correction should not be confused with that stated in Introduction.)

\section{EQUATIONS FOR THE FREE ENERGY CALCULATION}

In the previous study, ${ }^{12}$ the free energy of the system, $\Delta G$, was estimated by the following equations;

$$
\begin{gathered}
\Delta G / R T=C_{\mathrm{c}}\left(\ln C_{\mathrm{c}}+\ln \gamma_{\mathrm{c}}\right)+C_{\mathrm{b}} \ln \gamma_{\mathrm{b}} \\
+C_{\mathrm{p}} \ln \gamma_{\mathrm{p}}\left(=F_{\mathrm{ex}} / R T\right)+C_{\mathrm{w}} \ln a_{\mathrm{w}} \\
C_{\mathrm{c}}=C_{\mathrm{s}}+(1-v \theta) C_{\mathrm{p}} / v \\
C_{\mathrm{b}}=v C_{\mathrm{s}} \\
a_{\mathrm{w}}=C_{\mathrm{w}} /\left(C_{\mathrm{w}}+(1-v \theta) C_{\mathrm{p}} / v+(1+v) C_{\mathrm{s}}\right) \\
F_{\mathrm{ex}} / R T=C_{\mathrm{p}} \xi(1-v \theta)^{2} \sum_{m=1}^{N-1}(1-m / N) \\
\times \exp (-\kappa m b) / m \\
\kappa^{2}=8 \pi \times 10^{-3} N_{\mathrm{Av}}\left(e^{2} / D k T\right) I \\
I=\left(v^{2} C_{\mathrm{s}}+v C_{\mathrm{s}}+v(1-v \theta) C_{\mathrm{p}}\right) / 2 \\
\ln \gamma_{\mathrm{c}, \mathrm{b}}=\left(\partial\left(F_{\mathrm{ex}} / R T\right) / \partial C_{\mathrm{c}, \mathrm{b}}\right) C_{\mathrm{b}, \mathrm{c}}, C_{\mathrm{p}}, \theta \\
\ln \gamma_{\mathrm{p}}=\left(\partial\left(F_{\mathrm{ex}} / R T\right) / \partial C_{\mathrm{p}}\right)_{C_{\mathrm{c}}, C_{\mathrm{b}}, \theta}
\end{gathered}
$$

where $C$ means molar concentration and $a$ means activity. Subscripts $c, b, p, w$, and $s$ mean counterion, byion, polyion, water (solvent) and added salt, respectively. Excess free energy of polyion, $F_{\text {ex }}$, is estimated by sum- ming the electrostatic energy between charges on the polyion via the Debye-Hückel screened Coulombic potential as eq $4 \mathrm{a}-4 \mathrm{c}$. In the equation, $N_{\mathrm{Av}}$ is the Avogadro number and the ionic strength $I$ contains a contribution of "free" counterions of the polyion.

In the present paper, we correct eq 2 as eq 7 .

$\Delta G / R T=C_{\mathrm{c}} \ln C_{\mathrm{c}}+F_{\mathrm{ex}} / R T+C_{\mathrm{w}} \ln a_{\mathrm{w}}$

In this equation, $\ln \gamma_{c, b}$ terms are removed from eq 2. This is because the contribution to $\Delta G$ is already contained in $F_{\mathrm{ex}} / R T\left(=C_{\mathrm{p}} \ln \gamma_{\mathrm{p}}\right)$. This was called to the authors' attention by $K$. Iwasa of the National Institute of Health in Maryland. ${ }^{19}$ The correction leads to increases in the estimated $\theta$ values. However, in the qualitative sense, results and discussions given in the previous paper are valid as shown below. Further, we modify eq 7 based on the present model.

$$
\begin{aligned}
\Delta G / R T= & C_{\mathrm{c}}\left(\ln C_{\mathrm{c}}+\ln \gamma_{\mathrm{c}}{ }^{\mathrm{ii}}\right) \\
& +F_{\mathrm{ex}} / R T+C_{\mathrm{w}} \ln a_{\mathrm{w}}+C_{\mathrm{p}}(\theta \ln \theta \\
& +(1-v \theta) \ln (1-v \theta) \\
& -(1-(v-1) \theta) \ln (1-(v-1) \theta))
\end{aligned}
$$

where $\gamma_{c}$ ii the activity coefficient for the small ion-small ion interaction, is introduced according to the assumption (6). We estimate $\gamma_{c}{ }^{i i}$ by the extended Debye-Hückel equation of Kielland; ${ }^{20}$

$$
-\ln \gamma_{\mathrm{c}}{ }^{\mathrm{ii}}=1.178 v^{2} I_{\mathrm{o}}^{0.5} /\left(1+0.3291 r I_{\mathrm{o}}^{0.5}\right)
$$

where $I_{\mathrm{o}}=v(1+v) C_{\mathrm{s}} / 2$ and $r$ is the effective ion diameter given by Kielland. The last term of eq 8 expresses the mixing entropy for the linear array of free polymer charged sites and $v$ contiguous charged sites occupied by a bound counterion, introduced according to the assumption (2). The derivation is the same as that given for the charged site mixing on a polymer chain considered for the $\mathrm{pH}$ titration of weak polyacids. ${ }^{21} F_{\text {ex }} / R T$ in eq 8 (and also that in eq 7) is given by eq $4 a$. In the equation, 
the electrostatic free energy change due to the counterion condensation is expressed as caused by the reduction of the effective charge (i.e., $1-v \theta$ term). Here lies a difference of IMM and so-called site binding model. Based upon the proper site binding model, it should be expressed based on a change in the average charge spacing, $b$, because the latter model assumes complete neutralization of polymer charges by the counterion binding.

In the following discussion, we abbreviate the models according to eq 2, 7, 8 as PM (Previous Model), CPM (Corrected Previous Model) and IMM (Intermediate Model), respectively.

\section{EXPERIMENTAL}

\section{Materials}

Sodium polystyrenesulfonate (PSSNa) was obtained by polymerization of the monomer and purified by reprecipitation in ethyl alcohol. The viscosity averaged molecular weight, $\bar{M}_{v}$, was $9.3 \times 10^{4}$ (charge number, $N=452$ ). Sodium poly(L-glutamate) (PLGNa) was obtained by saponification of $\operatorname{poly}(\gamma-$ methyl L-glutamate) (AJICOAT-2000) kindly supplied by Ajinomoto Co. $\bar{M}_{v}$ was estimated as $6.9 \times 10^{4}(N=457)$. Polyarylamine hydrochloride (PAAHCl $(100 \%)$, where the percentage means the degree of neutralization) was purchased from Nittobo Co. $\bar{M}_{v}$ was $6.0 \times 10^{4}$ $(N=641)$. All the samples were purified by ultrafiltration through Ultrafilter UP-20 (Toyo Roshi Co.) and collected by freezedrying. The degree of neutralization of PAAHCl was adjusted by $\mathrm{NaOH}$ and $\mathrm{HCl}$, and the resultant $\mathrm{NaCl}$ was removed by the ultrafiltration. $\mathrm{NaCl}$ and $\mathrm{NaOH}$ were used as added salts; $\mathrm{NaOH}$ was used for PSSNa and PLGNa systems because $\mathrm{Na}$ ion selective electrode is disturbed by $\mathrm{H}^{+}$. However, the use of $\mathrm{NaCl}$ instead of $\mathrm{NaOH}$ exerted no appreciable influence on the measured $\gamma$ values at weakly acidic $\mathrm{pH}$ region $(\mathrm{pH}=5-6)$.

\section{Measurements}

Counterion activities were measured at $25 \pm 0.05^{\circ} \mathrm{C}$ by EMF method with ion-selective electrodes Orion 97-11 for $\mathrm{Na}^{+}$and Orion 9417 for $\mathrm{Cl}^{-}$in conjunction with an Orion 90-02 double junction reference electrode. The electrode potential was measured by an Orion 901 Ionanalyser. Activities of $\mathrm{Na}^{+}$and $\mathrm{Cl}^{-}$in the polymer-free solution for the calibration curves were estimated by the extended Debye-Hückel equation (eq 9). All sample solutions were prepared volumetrically with deionized and afterward distilled water.

\section{THEORETICAL PREDICTIONS OF CPM AND IMM FOR $\theta$ AND $\gamma$}

In Figures 1 and 2 the theoretical predictions of CPM and IMM for $\theta$ and $\gamma\left(=\gamma_{c}{ }^{\prime}\right.$ defined by eq 12) are compared. By referring to Figures 1 and 6 in our previous paper, ${ }^{12}$ it can be seen that for the dependences of $\theta$ and $\gamma$ on the ionic strength, the results of CPM qualita-

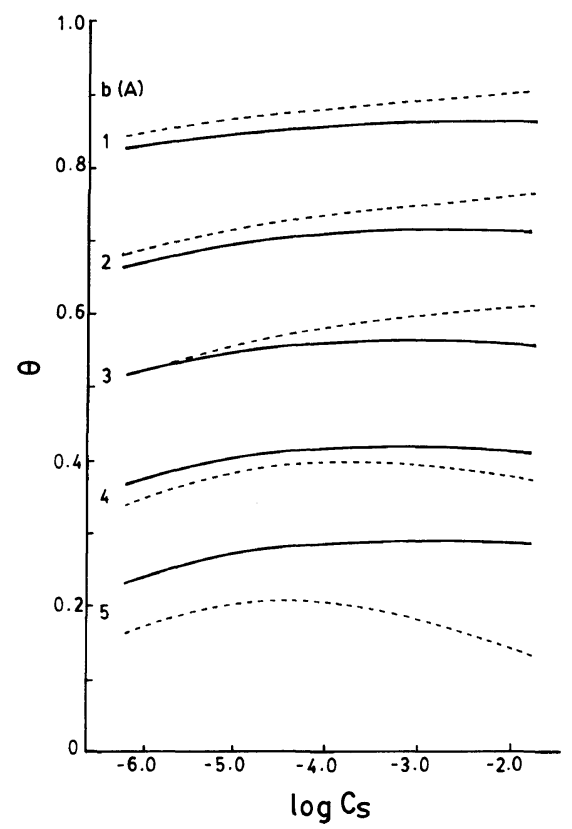

Figure 1. Comparison of the predictions for the dependence of $\theta$ on $C_{\mathrm{s}}$ by CPM (dashed lines) and IMM (thick lines). $N=1000, X=C_{\mathrm{p}} / C_{\mathrm{s}}=10^{-2}$. 


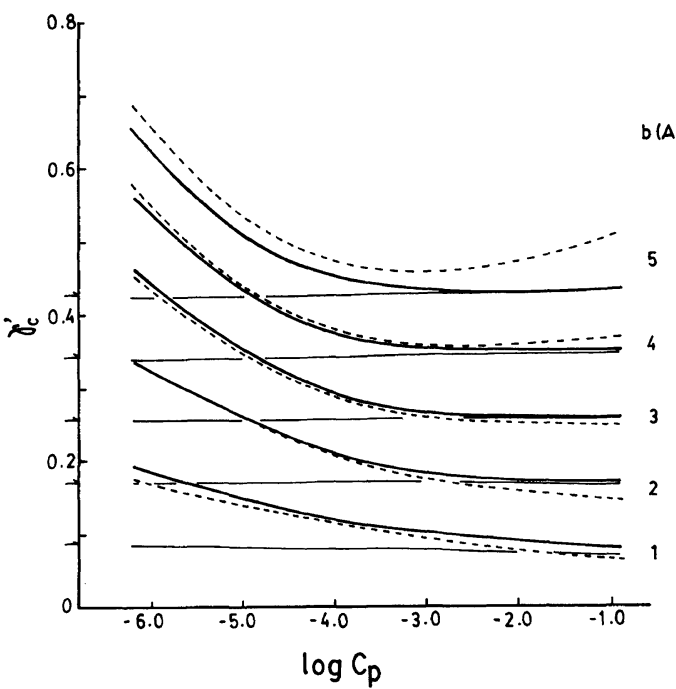

Figure 2. Comparison of the predictions for the dependence of $\gamma_{\mathrm{c}}{ }^{\prime}$ on $C_{\mathrm{p}}$ by CPM (dashed lines; $N=1000$ ) and IMM (thick lines; $N=1000$, thin lines; $N=\infty$ ). $C_{\mathrm{s}}=0 \mathrm{M}$. Arrows on the ordinate indicate the estimation by Manning theory.

tively agree with those of PM; both predict that $\theta$ values for polyions with low charge densities decrease under higher ionic strength and $\gamma$ values increase correspondingly. The dependence of $\theta$ on $N$ obtained by CPM is also essentially the same as that obtained by PM. Therefore, the previous discussions based on $\mathrm{PM}^{12}$ are also valid for CPM.

In Figure 1, it is seen that $\theta$ values estimated by IMM are lower than those of CPM for the higher charge densities $\left(b=1-3 \times 10^{-8} \mathrm{~cm}\right)$. However, the inverse is observed at the lower charge densities $\left(b=4-5 \times 10^{-8} \mathrm{~cm}\right)$. The corresponding bahaviors are found for the activity coefficients in Figure 2. This is because the last term in eq 8 makes a relatively unfavorable contribution to $\Delta G$ when $\theta$ value approaches 0 or 1. CPM, which lacks the entropy term, predicts a significant decrease in the effective charge density with the increase in the charge density, resulting in the unrealistic prediction on $\mathrm{p} K_{\mathrm{a}}$ values for weak polyacids. A detailed discussion on this point is presented in the subsequent paper (part II). ${ }^{14}$
Table I. $\theta$ values estimated by IMM, CPM, and Manning theory

\begin{tabular}{|c|c|c|c|c|}
\hline$v$ & $b\left(10^{-8} \mathrm{~cm}\right)$ & $\mathrm{IMM}^{\mathrm{a}}$ & $\mathrm{CPM}^{\mathrm{a}}$ & $\begin{array}{c}\text { Manning } \\
\text { theory }\end{array}$ \\
\hline \multirow[t]{7}{*}{1} & 1.0 & 0.847 & 0.867 & 0.860 \\
\hline & 2.0 & 0.697 & 0.718 & 0.720 \\
\hline & 3.0 & 0.549 & 0.557 & 0.580 \\
\hline & 4.0 & 0.406 & 0.385 & 0.440 \\
\hline & 5.0 & 0.273 & 0.205 & 0.300 \\
\hline & 10.0 & 0.006 & 0.0 & 0.0 \\
\hline & 20.0 & 0.0 & 0.0 & 0.0 \\
\hline \multirow[t]{7}{*}{2} & 1.0 & 0.455 & 0.465 & 0.465 \\
\hline & 2.0 & 0.411 & 0.425 & 0.430 \\
\hline & 3.0 & 0.367 & 0.380 & 0.395 \\
\hline & 4.0 & 0.323 & 0.332 & 0.360 \\
\hline & 5.0 & 0.280 & 0.281 & 0.325 \\
\hline & 10.0 & 0.086 & 0.0 & 0.150 \\
\hline & 20.0 & 0.001 & 0.0 & 0.0 \\
\hline \multirow[t]{7}{*}{3} & 1.0 & 0.310 & 0.317 & 0.318 \\
\hline & 2.0 & 0.289 & 0.298 & 0.302 \\
\hline & 3.0 & 0.267 & 0.277 & 0.287 \\
\hline & 4.0 & 0.245 & 0.254 & 0.271 \\
\hline & 5.0 & 0.223 & 0.229 & 0.256 \\
\hline & 10.0 & 0.116 & 0.088 & 0.178 \\
\hline & 20.0 & 0.005 & 0.0 & 0.022 \\
\hline
\end{tabular}

${ }^{\mathrm{a}} N=1000, C_{\mathrm{s}}=1 \times 10^{-5} \mathrm{M}$ and $C_{\mathrm{p}}=1 \times 10^{-7}$ eq. $/ \mathrm{l}$.

In Table I, $\theta$ values obtained by these theories are compared for $v=1-3$ and $b=1-20 \times 10^{-8} \mathrm{~cm}$. As seen in the table, the $\theta$ values estimated by IMM are lower than those by Manning theory. This may be because the estimation of the mixing entropy of the condensed counterions in IMM is different from that in Manning theory, namely, $\theta \ln \left(\theta / V_{\mathrm{p}}\right)$.

One can find another marked feature of IMM in that even at very low charge densities, the model predicts counterion binding to occur; for most cases with $\xi$ larger than the critical charge density of Manning theory $\left(v^{-1}\right)$, both of IMM and CPM estimate smaller $\theta$ values than Manning theory, while for $\xi<v^{-1}$ only IMM estimates non-zero values for $\theta$. This appears to reflect the site bindinglike character of IMM, invested by the last term of eq 8 .

In Table II, a comparison of IMM, CPM, 
Table II. $\gamma_{c}^{\prime}$ estimated by IMM, CPM, and theories of Manning and Iwasa et al

\begin{tabular}{cccccc}
\hline$b\left(10^{-8} \mathrm{~cm}\right)$ & $\mathrm{X}^{\mathrm{a}}$ & \multicolumn{1}{c}{$\mathrm{IMM}^{\mathrm{b}}$} & $\mathrm{CPM}^{\mathrm{c}}$ & Manning & \\
\hline \multirow{2}{*}{1.0} & 0.1 & 0.919 & 0.916 & 0.919 & 0.916 \\
& 0.5 & 0.701 & 0.695 & 0.701 & 0.692 \\
& 1.0 & 0.552 & 0.544 & 0.552 & 0.539 \\
& 5.0 & 0.254 & 0.243 & 0.249 & 0.231 \\
& $\infty$ & 0.100 & 0.090 & 0.085 & 0.085 \\
& $(\infty$ & 0.153 & 0.139 & 0.085 & $0.085)$ \\
3.0 & 0.1 & 0.939 & 0.938 & 0.938 & 0.930 \\
& 0.5 & 0.773 & 0.771 & 0.769 & 0.744 \\
& 1.0 & 0.657 & 0.652 & 0.651 & 0.616 \\
& 5.0 & 0.409 & 0.404 & 0.400 & 0.363 \\
& $\infty$ & 0.268 & 0.263 & 0.255 & 0.255 \\
5.0 & $(\infty$ & 0.351 & 0.348 & 0.255 & $0.255)$ \\
& 0.1 & 0.958 & 0.964 & 0.956 & 0.944 \\
& 0.5 & 0.840 & 0.856 & 0.835 & 0.795 \\
& 1.0 & 0.752 & 0.771 & 0.747 & 0.693 \\
& 5.0 & 0.553 & 0.578 & 0.546 & 0.499 \\
& $\infty$ & 0.434 & 0.462 & 0.425 & 0.425 \\
& $\infty$ & 0.505 & 0.529 & 0.425 & $0.425)$ \\
\hline
\end{tabular}

a $X=C_{\mathrm{p}} / C_{\mathrm{s}}, C_{\mathrm{p}}=1 \times 10^{-3}$ eq. $/ \mathrm{l}$, for the parenthesized values $C_{\mathrm{p}}=1 \times 10^{-5}$ eq. $/ 1 . \quad$ b eq 12 with eq $8 . \quad{ }^{\text {c }}$ eq 12 with eq $7 .^{\mathrm{d}}$ eq 10 . $^{\mathrm{e}}$ eq 11 .

and theories of Manning ${ }^{1}$ and Iwasa et al..$^{22}$ is given for counterion activity coefficients in the presence of added salt, namely, $\gamma_{\mathrm{c}}{ }^{\prime}$, for $\xi>1$ and $v=1$. These are estimated by the following equations;

$$
\begin{aligned}
& \gamma_{c}{ }^{\prime}=\left(\xi^{-1} X+1\right)(X+1)^{-1} \\
& \exp \left[-0.5 \xi^{-1} X /\left(\xi^{-1} X+2\right)\right] \\
& \gamma_{c}{ }^{\prime}=\left(\xi^{-1} X+1\right)(X+1)^{-1} \\
& \exp \left[(-0.5-0.3906) \xi^{-1} X /\left(\xi^{-1} X+2\right)\right. \\
& \left.+0.3906\left(\xi^{-1} X /\left(\xi^{-1} X+2\right)\right)^{2}\right] \\
& \gamma_{c}{ }^{\prime}=[(1-\theta) X+1](X+1)^{-1} \gamma_{c}
\end{aligned}
$$

where $X=C_{\mathrm{p}} / C_{\mathrm{s}}$. Equations 10 and 11 were those derived by Manning ${ }^{1}$ and Iwasa et al., ${ }^{2}$ respectively. The latter contains the contribution of the higher order cluster terms. Equation 12 is used for IMM and CPM. Here $\gamma_{c}$ is the activity coefficient caused by the electrostatic interactions while $\gamma_{c}^{\prime}$ contains an additional term to include the contribution of a fraction of free counterions. $\gamma_{c}$ is given by eq 5 , and $\theta$ 's of eq 5 and eq 12 are given by eq 7 for CPM and by eq 8 for IMM. As seen in Table II, $\gamma_{c}{ }^{\prime}$ estimated by IMM and CPM agrees with those by Manning better than by Iwasa et al., which estimates stronger interaction between uncondensed counterions and the polyion than Manning theory. However, a significant discrepancy between $\gamma_{c}{ }^{\prime}$ values by eq 12 (IMM and CPM) and eq 10 and 11 (Manning and Iwasa et al.) is observed at lower ionic strength as exemplified by the parenthesized values in Table II. This is because IMM and CPM predict a marked increase of $\gamma_{c}{ }^{\prime}$ with decreasing $C_{p}$ in the absence of added salt (Figure 2). Such a dependence of $\gamma_{\mathrm{c}}{ }^{\prime}$ on $C_{\mathrm{p}}$ has been experimentally observed as shown in Section of comparison with experimental data on $\gamma_{c}{ }^{\prime}$. One would take the above result as suggesting that the formulations used in IMM and CPM are essentially different from that of Manning theory. However, this is not the case. The origin of the discrepancy is not in the formalism but in the charge number $N$ ! In Figure 2 the $C_{\mathrm{p}}$ dependence of $\gamma_{\mathrm{c}}{ }^{\prime}$ estimated by IMM for $N=\infty$ is shown. (The dependence estimated by CPM is essentially the same as that by IMM.) The theoretical curves were obtained by using the following expression for $F_{\text {ex }} / R T$;

$$
F_{\mathrm{ex}} / R T=-C_{\mathrm{p}} \xi(1-v \theta)^{2} \ln \left(1-\mathrm{e}^{-\kappa b}\right)
$$

This equation is the same as that used in Manning's two phase model. ${ }^{2}$ Figure 2 demonstrates that at $N=\infty$ IMM estimates $\gamma_{c}{ }^{\prime}$ values which are hardly dependent on $C_{\mathrm{p}}$ and are very close to those by eq 10 and 11 as indicated by the arrows on the ordinate. The striking effect on $\gamma_{c}{ }^{\prime}$ of the extrapolation to $N=\infty$ suggests that a polymer model, of which $N$ is finite, should be used to study ionic strength dependence of the counterion condensation phenomenon for real polyelectrolytes. Bratko and Dolar, ${ }^{23}$ too, have recently reported that based on the Poisson- 
Boltzmann equation, osmotic coefficients of polyelectrolyte solution are least dependent on $C_{\mathrm{p}}$ at $N=\infty$. On the other hand, Soumpasis $^{24}$ concluded based on a Debye-Hückel theory that in the limit of infinite dilution extended polyions are satisfactorily represented by Manning's model. However in his study the end effects were neglected; the conclusion should be applied to hypothetical polyions with infinite length. This is because in the limit of infinite dilution the counterion condensation does not occur for polyions of finite length, as shown by Woodbury and Ramanathan. ${ }^{25}$ Thus, for high dilute systems of real polyion, theories based on infinitely long polymer model are inadequate to treat the condensation phenomena. It should be noted from comparison of the theoretical curves for $N=1000$ and $\infty$ in Figure 2 that at $C_{\mathrm{p}}$ region usually studied $\left(10^{-4}-10^{-1} \mathrm{M}\right)$ the effect of the polymer length becomes much less remarkable and the estimations by eq 10 and 11 agree with those by eq 12 .

\section{COMPARISON WITH EXPERIMENTAL DATA ON $\gamma_{c}{ }^{\prime}$}

In our previous study, ${ }^{12}$ we compared counterion activity coefficients measured for several polymer systems with the theoretical predictions by our previous model (PM). We found that PM could qualitatively explain the data reported by Kowblansky et al. ${ }^{26}$ According to their study, $\gamma_{c}{ }^{\prime}$ of sodium polyphosphate (PPNa, the axial charge spacing $b=2.55 \times$ $10^{-8} \mathrm{~cm}$ ) slightly decreases with increasing the ionic strength, whereas $\gamma_{c}{ }^{\prime}$ of sodium carrageenan (CarrNa, $\left.b=4.463 \times 10^{-8} \mathrm{~cm}\right)$ significantly increases. These facts suggest that the ionic strength dependence of $\gamma_{c}{ }^{\prime}$ is largely dependent on the charge density of polyions, although such a dependence of $\gamma_{c}{ }^{\prime}$ has not been found in other polyion systems, to the authors' knowledge.

On Table III listed are experimental counterion activity coefficients divided by $\gamma_{c}{ }^{i i}$, $\gamma_{c}{ }^{\mathbf{e x}} / \gamma_{c}{ }^{i i}$, obtained in the present study for the polyelectrolyte systems at $X=4-5$ and $C_{\mathrm{p}}=5 \times 10^{-4}-1 \times 10^{-2}$ eq $1^{-1}$ with $\gamma_{\mathrm{c}}{ }^{\prime}$ values $\left(\equiv \gamma_{c}{ }^{e x} / \gamma_{c}{ }^{i i}\right)$ calculated by IMM and CPM. For polymers with relatively high charge densities, i.e., PSSNa, PLGNa and PAAHCl $(100 \%)$, experimental $\gamma_{c}{ }^{\prime}$ values decrease with the increase of the ionic strength, which both IMM and CPM qualitatively predict. However, the decrease in the theoretical $\gamma_{c}{ }^{\prime}$ values is much smaller than the experimental values. At lower charge densities too, the predicted trends of $\gamma_{c}{ }^{\prime}$ by IMM and CPM are consistent with those of the experimental results; at $C_{\mathrm{p}}$ between $5 \times 10^{-3}-1 \times 10^{-2}$ eq $1^{-1}, \gamma_{c}{ }^{\prime}$ of $\mathrm{PAAHCl}$ $(60 \%)$ and PAAHCl $(50 \%)$ are almost constant or slightly increase. As shown in Figure 4 of ref 25 , CarrNa exhibits considerably large increase of $\gamma_{c}{ }^{\prime}$ with the ionic strength. The charge density of CarrNa was between those of PAAHCl $(60 \%)$ and PAAHCl $(50 \%)$. Therefore it does not seem that the charge density is a single factor that determines qualitative and quantitative behavior of the ionic strength dependence of $\gamma_{c}{ }^{\prime}$. Other characteristics which are not considered in our models may take a part in the behavior of $\gamma_{c}{ }^{\prime}$. Diakun et al. ${ }^{27}$ observed that $\gamma_{c}{ }^{\prime}$ of sodium heparinates in aqueous $\mathrm{NaCl}$ solutions increase with increasing the ionic strength for the polyions with $b=2.9-4.3 \times 10^{-8} \mathrm{~cm}$ and ascribed the dependence to a contracted conformation at higher $C_{\mathrm{p}}$.

In the case of salt-free solutions too, a similar trend of $\gamma_{c}{ }^{\prime}$ as in the presence of added salts is observed as shown in Figure 3. For polyions of relatively low charge densities $(\mathrm{PAAHCl}(50 \%$ and $60 \%)), \gamma_{\mathrm{c}}{ }^{\prime}$ values take a minimum or increase with increasing $C_{\mathrm{p}}$. For polyions of higher charge densities (PAAHCl $(100 \%)$, PSSNa, PLGNa), $\gamma_{c}{ }^{\prime}$ values hardly depend on $C_{\mathrm{p}}$ or decrease with increasing $C_{\mathrm{p}}$ in the $C_{\mathrm{p}}$ region studied. Thus with these results alone, one may conclude that the dependency of $\gamma_{c}{ }^{\prime}$ on the ionic strength changes with the charge density. However experimental data 
Table III. Comparison of experimental and theoretical $\gamma_{c}^{\prime}$ values

\begin{tabular}{|c|c|c|c|c|c|c|}
\hline \multirow{2}{*}{ Polymer } & \multirow{2}{*}{$\begin{array}{c}b \\
\left(10^{-8} \mathrm{~cm}\right)\end{array}$} & \multirow{2}{*}{$\begin{array}{c}X \\
\left(C_{\mathrm{p}} / C_{\mathrm{s}}\right)\end{array}$} & \multirow{2}{*}{$\begin{array}{c}C_{\mathrm{p}} \\
\left(10^{-3} \mathrm{M}\right)\end{array}$} & \multirow{2}{*}{$\begin{array}{c}\text { Exptl. } \\
\left(\gamma_{c}^{\text {ex }} / \gamma_{c}^{i i}\right)\end{array}$} & \multicolumn{2}{|c|}{$\gamma_{c}^{\prime}$} \\
\hline & & & & & IMM & CPM \\
\hline \multirow[t]{3}{*}{$\mathrm{PSSNa}^{\mathrm{a}}$} & 2.5 & 4.43 & 0.557 & 0.465 & 0.398 & 0.390 \\
\hline & & & 1.11 & 0.429 & 0.393 & 0.384 \\
\hline & & & 11.1 & 0.403 & 0.385 & 0.372 \\
\hline \multirow{2}{*}{ PLGNa $^{\mathrm{b}}$} & 3.6 & 4.91 & 0.987 & 0.451 & 0.463 & 0.465 \\
\hline & & & 9.87 & 0.400 & 0.458 & 0.458 \\
\hline \multirow[t]{3}{*}{$\operatorname{PAAHCl}(100 \%)^{\mathrm{c}}$} & 2.5 & 4.23 & 0.531 & 0.470 & 0.398 & 0.390 \\
\hline & & & 1.06 & 0.426 & 0.395 & 0.386 \\
\hline & & & 10.6 & 0.374 & 0.390 & 0.377 \\
\hline \multirow[t]{3}{*}{$\operatorname{PAAHCl}\left(60^{\circ}\right)^{\mathrm{d}}$} & 4.1 & 4.09 & 1.03 & 0.580 & 0.521 & 0.529 \\
\hline & & & 5.14 & 0.520 & 0.515 & 0.525 \\
\hline & & & 10.3 & 0.516 & 0.516 & 0.526 \\
\hline \multirow[t]{2}{*}{$\operatorname{PAAHCl}(50 \%)^{\mathbf{e}}$} & 5.0 & 4.00 & 5.03 & 0.576 & 0.580 & 0.612 \\
\hline & & & 10.1 & 0.578 & 0.580 & 0.616 \\
\hline
\end{tabular}

a $N=452$, in $\mathrm{NaOH} .{ }^{\text {b }} N=457$, in $\mathrm{NaOH} .{ }^{\text {c }} N=641$, in NaCl. ${ }^{\text {d }} N=387$, in NaCl. $\quad$ e $N=320$, in NaCl.

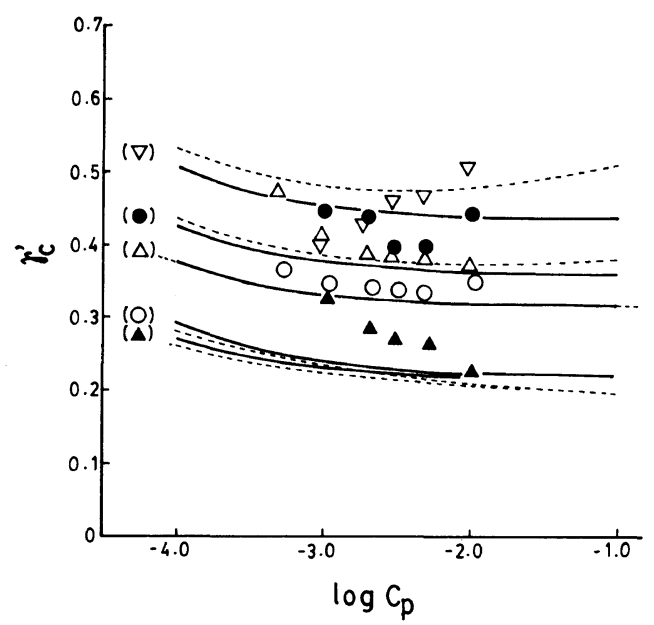

Figure 3. Comparison of $\gamma_{c}^{\prime}$ for various polyelectrolyte systems with the theoretical curves by CPM and IMM. (O) PSSNa, $N=452, b=2.5 \times 10^{-8} \mathrm{~cm}$. ( $\triangle$ ) PLGNa, $N=457, b=3.6 \times 10^{-8} \mathrm{~cm}$. (A) PAAHCl $(100 \%), \quad N=641, \quad b=2.5 \times 10^{-8} \mathrm{~cm}$. (O) PAAHCl $(60 \%), N=387, b=4.1 \times 10^{-8} \mathrm{~cm} .(\nabla) \operatorname{PAAHCl}(50 \%)$, $N=320, b=5.0 \times 10^{-8} \mathrm{~cm}$. Dashed curves, CPM; thick curves, IMM; from bottom to top, PAAHCl $(100 \%)$, PSSNa, PLGNa, PAAHCl (60\%), PAAHCl (50\%).

incompatible with this simple interpretation have been reported. ${ }^{28-30}$ Figure 6 of our previous paper ${ }^{12}$ collects $\gamma_{c}{ }^{\prime}$ for sodium poly- (vinyl alcohol sulfate) (PVSNa, $b=3.38 \times$ $10^{-8}$ and $\left.5.8 \times 10^{-8} \mathrm{~cm}\right),{ }^{28}$ silver salt of carboxymethylcellulose (CMCAg, $b=3.31 \times$ $\left.10^{-8} \mathrm{~cm}\right),{ }^{29}$ and sodium poly(methylstyrenesulfonate) (PMSSNa, $\left.b=2.66 \times 10^{-8} \mathrm{~cm}\right){ }^{30}$ In the figure, the followings can be seen; 1) $\gamma_{c}{ }^{\prime}$ of PVSNa increases with the increase of $C_{\mathrm{p}}$ for the two charge densities. 2) At the same $C_{\mathrm{p}}$ region as for PVSNa, $\gamma_{\mathrm{c}}{ }^{\prime}$ of $\mathrm{CMCAg}$ decreases with the increase of $C_{p}$. 3) PMSSNa takes a distinct minimum of $\gamma_{c}{ }^{\prime}$ at $C_{\mathrm{p}} \simeq 10^{-3}$ eq $1^{-1}$, whereas $\gamma_{\mathrm{c}}{ }^{\prime}$ of PSSNa with a similar charge density are least dependent on $C_{\mathrm{p}}$ as observed in this study. These experimental facts suggest that each $\gamma_{c}{ }^{\prime}$ in the absence of added salts takes a minimum, irrespective of the charge density, at a $C_{\mathrm{p}}$, which changes from a polyion to another. In other words, $\gamma_{\mathrm{c}}{ }^{\prime}$ decreases in a lower $C_{\mathrm{p}}$ region and increases in a higher $C_{\mathrm{p}}$ region with increasing $C_{\mathrm{p}}$. If we confine our discussion to the lower $C_{\mathrm{p}}$ region, both IMM and CPM can predict the decreasing tendency of $\gamma_{\mathrm{c}}{ }^{\prime}$ with increasing $C_{\mathrm{p}}$. The trend has been also confirmed by other experimental techniques. In studies on the conductivity ${ }^{31,32}$ and on the 
self diffusion coefficient ${ }^{33,34}$ of counterions in polyelectrolyte solutions, it has been observed that both experimental data decrease with increasing $C_{\mathrm{p}}$ for $C_{\mathrm{p}} \leqq 0.01 \mathrm{eq} \mathrm{1}^{-1}$. The qualitative agreement of the predictions on $\gamma_{c}{ }^{\prime}$ by IMM and CPM with the experimental observation in the $C_{\mathrm{p}}$ region seems not to be fortuitous because it is in the lower $C_{\mathrm{p}}$ region that the assumptions in the models are verified; in the dilute polymer solutions, namely $c a .<0.01$ eq $1^{-1}$, the crossover of the polymer chains is not significant, and hence interpolymer interactions is neglected.

However IMM and CPM can not predict the increasing trend of observed $\gamma_{c}{ }^{\prime}$ values, irrespective of the charge density, in the higher polymer concentration range. One possible reason for this may be the overlapping of the polymer chains. In a limit of the crossover of the polymer chains, ${ }^{35}$ distance between polymer chains becomes so close $\left(e . g ., \ll \kappa^{-1}\right)$ that the two phases defined in the condensation theories will becomes diffuse, whereby condensed counterions and free ions are not to be distinguished. In the higher $C_{\mathrm{p}}$ region, in other words, the counterion condensation phenomenon itself is fading. Naturally in the presence of added salts, such a trend will be less appreciable because of the smaller ionic atmosphere, $\kappa^{-1}$. This is consistent with the experimental data hitherto obtained, including those of present study.

In summary, we proposed a new model of counterion condensation as an extention of Manning's two phase model and compared $\theta$ and $\gamma$ values predicted by the models, IMM and CPM, with those by Manning and Iwasa et al. The results showed that infinitely long polymer-chain model is not appropriate to treat the ionic strength dependence of the condensation phenomena in real polyion systems.

In the comparison with the experimental data on $\gamma_{c}{ }^{\prime}$, IMM and CPM can predict the decreasing trend with the increase in $C_{\mathrm{p}}$ in the dilute solutions, but not the increasing ten- dency of $\gamma_{\mathrm{c}}{ }^{\prime}$ in the higher $C_{\mathrm{p}}$ region. The latter may be attributed to the crossover of polymer chains.

In the subsequent paper (Part II), ${ }^{14}$ IMM and CPM are applied to the potentiometric titration of weak polyacids. A significant difference between IMM and CPM will be shown in the study.

Acknowledgement. We are grateful to Dr. K. Iwasa for his valuable discussions on the correction of our previous model and the encouragement for the publication of the present paper.

\section{REFERENCES}

1. G. S. Manning, J. Chem. Phys., 51, 924 (1969).

2. G. S. Manning, Q. Rev. Biophys., 11, 179 (1978).

3. M. T. Record, Jr., C. F. Anderson, and T. M. Lohman, Q. Rev. Biophys., 11, 103 (1978).

4. N. Ise and T. Okubo, Macromolecules, 11, 439 (1978).

5. Y. M. Joshi and J. C. T. Kwak, Biophys. Chem., 13, 65 (1981).

6. J. Mattai and J. C. T. Kwak, Macromolecules, 19 , 1663 (1986).

7. G. S. Manning, Biopolymers, 23, 2671 (1984).

8. S. Miyamoto, Biophys. Chem., 9, 79 (1979).

9. H. Daoust and M. A. Chabot, Macromolecules, 13, 616 (1980).

10. K. W. Rhee and B. R. Ware, J. Chem. Phys., 78, 3349 (1983).

11. G. S. Manning, J. Phys. Chem., 88, 6654 (1984).

12. M. Satoh, J. Komiyama, and T. Iijima, Macromolecules, 18, 1195 (1985).

13. M. Satoh, T. Kawashima, J. Komiyama, and J. Iijima, Biophys. Chem., to be submitted.

14. M. Satoh and J. Komiyama, Polym. J., 19, 1201 (1987).

15. F. E. Harris and S. A. Rice, J. Phys. Chem., 58, 725 (1954).

16. S. Sasaki and A. Minakata, Biophys. Chem., 11, 199 (1980).

17. J. D. Wells, Proc. R. Soc. London, Ser. B., 183, 399 (1973).

18. Y. M. Joshi and J. C. T. Kwak, J. Phys. Chem., 83, 1978 (1979).

19. private communications.

20. J. Kielland, J. Am. Chem. Soc., 59, 1675 (1937).

21. G. S. Manning, J. Phys. Chem. 85, 870 (1981).

22. K. Iwasa and J. C. T. Kwak, J. Phys. Chem., 81, 408 (1977). 
23. D. Bratko and D. Dolar, J. Chem. Phys., 80, 5782 (1984).

24. D. Soumpasis, J. Chem. Phys., 69, 3190 (1978).

25. C. P. Woodbury, Jr. and G. V. Ramanathan, Macromolecules, 15, 82 (1982).

26. M. Kowblansky, M. Tomasula, and P. Ander, $J$. Phys. Chem., 82, 1491 (1978).

27. G. P. Diakun, H. E. Edwards, D. J. Wedlock, J. C. Allen, and G. O. Phillips, Macromolecules, 11, 1110 (1978).

28. M. Nagasawa and I. Kagawa, J. Polym. Sci., 25, 61 (1957).
29. I. Kagawa and K. Katsuura, J. Polym. Sci., 17, 365 (1955).

30. S. Oman and D. Dolar, Z. Phys. Chem., (Frankfurt/Main) 56, 1 (1967).

31. J. C. T. Kwak, G. F. Murphy, and E. J. Spiro, Biophys. Chem., 7, 379 (1978).

32. S. Miyamoto, Biophys. Chem., 9, 79 (1979).

33. R. Fernandez-Prini, E. Baumgartner, S. Liberman, and A. E. Lagos, J. Phys. Chem., 73, 1420 (1969).

34. W. Lubas and P. Ander, Macromolecules, 13, 318 (1980).

35. M. Mandel, Eur. Polym. J., 19, 911 (1983). 